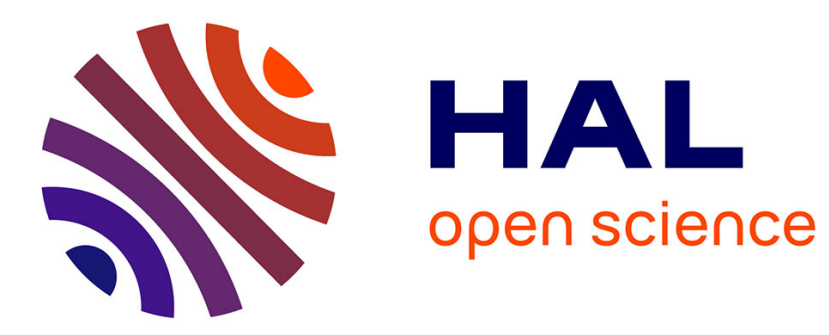

\title{
Numerical study of unsteady rarefied gas flow through an orifice
}

\author{
M.T. T Ho, Irina Martin Graur
}

\section{To cite this version:}

M.T. T Ho, Irina Martin Graur. Numerical study of unsteady rarefied gas flow through an orifice. Vacuum, 2014, 109, pp.253 - 265. 10.1016/j.vacuum.2014.05.004 . hal-01784154

\section{HAL Id: hal-01784154 \\ https://hal.science/hal-01784154}

Submitted on 22 May 2018

HAL is a multi-disciplinary open access archive for the deposit and dissemination of scientific research documents, whether they are published or not. The documents may come from teaching and research institutions in France or abroad, or from public or private research centers.
L'archive ouverte pluridisciplinaire HAL, est destinée au dépôt et à la diffusion de documents scientifiques de niveau recherche, publiés ou non, émanant des établissements d'enseignement et de recherche français ou étrangers, des laboratoires publics ou privés. 


\section{- Numerical study of unsteady rarefied gas flow through an

5 Abstract

Transient flow of rarefied gas through an orifice caused by various pressure ratios between the reservoirs is investigated for a wide range of the gas rarefaction, varying from the free molecular to continuum regime. The problem is studied on the basis of the numerical solution of unsteady S-model kinetic equation. It is found that the mass flow rate takes from 2.35 to 30.37 characteristic times, which is defined by orifice radius over the most probable molecular speed, to reach its steady state value. The time of steady flow establishment and the steady state distribution of the flow parameters are compared with previously reported data obtained by the Direct Simulation Monte Carlo (DSMC) method. A simple fitting expression is proposed for the approximation of the mass flow rate evolution in time.

- Keywords: rarefied gas, kinetic equation, orifice, transient flow

\section{1. Introduction}

8

The nonequilibrium flows of gases appear in different technological domains like the vacuum equipment, high altitude aerodynamics and in a relatively new field as the microelectromechanical systems (MEMS). The deviation of a gas from its local equilibrium state can be characterized by the Knudsen number, which present the ratio between the molecular mean free path and the characteristic length of the problem. For the relatively large values of the Knudsen number the classical continuum approach fails to describe the gas behavior and the kinetic equations, like the Boltzmann equation or model kinetic equations, must be solved to simulate the gas flows.

The gas flow through a thin orifice is a problem of a large practical interest for the design of the vacuum equipment, space or the microfluidic applications. The under-expanded jets through the orifices are predominately used by particle analyzer systems to separate and isolate molecules, ions of substances for analyzing their physical and chemical properties. The time dependent characteristics of these jets are important for the investigation of the response time of the vacuum gauges developed for the measurements of the rapid pressure changes [1].

Email addresses: mtho@polytech.univ-mrs.fr (M.T. Ho), irina.martin@univ-amu.fr 
The steady state flows through the orifice, slit and short tube have been successfully studied applying the DSMC method and the kinetic equations [2], [3], [4], [5], [6], [7], [8], [9]. However, only a few results on the transient rarefied flows through an orifice [10], a short tube [11], a long tube [12] or a slit [13] may be found in open literature. The flow conditions in [10] are limited to high and moderate Mach number owing to significant statical noise of DSMC method at low Mach number. The authors of [1] also studied experimentally and numerically the transient gas flow, but between two tanks of the fixed volumes. The rapid high amplitude pressure changings in time are examined and their characteristic time was found to be of the order of few seconds.

The aim of this work is to analyze the transient properties of gas flow through an orifice induced by various values of the pressure ratio over a broad range of gas rarefaction. The unsteady nonlinear S-model kinetic equation is solved numerically by Discrete Velocity Method (DVM) to obtain the mass flow rate and macroscopic parameters as a function of time. The time to reach the steady state conditions for the mass flow rate is also estimated. An empirical expression for evaluation of time-dependent mass flow rate is proposed.

\section{Problem formulation}

Consider an orifice of radius $R_{0}$ contained in an infinitesimally thin wall, which isolates two infinite reservoirs. Both the upstream and downstream reservoirs are filled with a monatomic gas but maintained at different pressures $p_{0}$ and $p_{1}$, respectively, with $p_{0}>p_{1}$. The temperatures of the wall and of the gas in the reservoirs are equal to $T_{0}$. At time $t=0$, the orifice is opened instantly and the gas starts to flow from the upstream reservoir to the downstream one.

Let us introduce a cylindrical coordinate system $\left(r^{\prime}, \vartheta, z^{\prime}\right)$ with the origin positioned at the center of the orifice and the $O z^{\prime}$ axis directed along the axis of the reservoirs (see the lateral section shown in Fig. 1). We assume that the flow is cylindrically symmetric and does not depend on the angle $\vartheta$ and therefore the problem may be considered as two dimensional in the physical space with the position vector $\boldsymbol{s}^{\prime}=\left(r^{\prime}, z^{\prime}\right)$.

The gas-surface interaction has a very small impact on an orifice flow [14]; consequently, this flow is governed by two principal parameters: the pressure ratio $p_{1} / p_{0}$ and gas rarefaction $\delta$ determined as

$$
\delta=\frac{R_{0} p_{0}}{\mu_{0} v_{0}}, \quad v_{0}=\sqrt{\frac{2 k T_{0}}{m}},
$$

where $\mu_{0}$ is the viscosity coefficient at the temperature $T_{0}, v_{0}$ is the most probable molecular speed at the same temperature; $m$ is the molecular mass of the gas; $k$ is the Boltzmann constant. It is to note that the gas rarefaction parameter is inversely proportional to the Knudsen number; i.e., when $\delta$ varies from 0 to $\infty$, the flow regime changes from the free molecular to the hydrodynamic regime.

It is convenient to define the characteristic time $t_{0}$ of the flow as follows 


$$
t_{0}=\frac{R_{0}}{v_{0}} .
$$

$$
\frac{\partial}{\partial t^{\prime}}\left(r^{\prime} f^{\prime}\right)+\frac{\partial}{\partial r^{\prime}}\left(r^{\prime} f^{\prime} v_{p} \cos \varphi\right)-\frac{\partial}{\partial \varphi}\left(f^{\prime} v_{p} \sin \varphi\right)+\frac{\partial}{\partial z^{\prime}}\left(r^{\prime} f^{\prime} v_{z}\right)=r^{\prime} \nu^{\prime}\left(f^{S^{\prime}}-f^{\prime}\right)
$$

$$
f^{S^{\prime}}=f^{M^{\prime}}\left[1+\frac{2 m \boldsymbol{V} \boldsymbol{q}^{\prime}}{15 n^{\prime}(k T)^{2}}\left(\frac{m V^{2}}{2 k T^{\prime}}-\frac{5}{2}\right)\right], f^{M^{\prime}}=n^{\prime}\left(\frac{m}{2 \pi k T^{\prime}}\right)^{3 / 2} \exp \left(-\frac{m V^{2}}{2 k T^{\prime}}\right),
$$

The unsteady S-model kinetic equation [15] is used to simulate the tranThe main unknown is the molecular velocity distribution function $f^{\prime}\left(t^{\prime}, \boldsymbol{s}^{\prime}, \boldsymbol{v}\right)$, $\boldsymbol{v}=\left(v_{p} \cos \varphi, v_{p} \sin \varphi, v_{z}\right)$ is the molecular velocity vector representing the molecular velocity space. The polar coordinates are introduced in a plane $\left(v_{r}, v_{\vartheta}\right)$ and $v_{p}, \varphi$ are the magnitude and orientation of the molecular velocity vector in this $\left(v_{r}, v_{\vartheta}\right)$ plane. The molecular collision frequency $\nu^{\prime}$ is supposed to be independent on the molecular velocity and can be evaluated [15] by

$$
\nu^{\prime}=\frac{p^{\prime}}{\mu^{\prime}} .
$$

where $f^{M^{\prime}}$ is the local Maxwellian distribution function, $\boldsymbol{V}=\boldsymbol{v}-\boldsymbol{u}^{\prime}$ is the peculiar velocity vector, $\boldsymbol{u}^{\prime}=\left(u_{r}^{\prime}, 0, u_{z}^{\prime}\right)$ is the bulk velocity vector, $\boldsymbol{q}^{\prime}=\left(q_{r}^{\prime}, 0, q_{z}^{\prime}\right)$ is the heat flux vector, $n^{\prime}$ is the gas numerical density.

It is useful to define the dimensionless variables as follows

$$
\begin{gathered}
t=\frac{t^{\prime}}{t_{0}}, \boldsymbol{s}=\frac{\boldsymbol{s}^{\prime}}{R_{0}}, \quad \boldsymbol{c}=\frac{\boldsymbol{v}}{v_{0}}, \boldsymbol{u}=\frac{\boldsymbol{u}^{\prime}}{v_{0}}, \quad n=\frac{n^{\prime}}{n_{0}}, \\
T=\frac{T^{\prime}}{T_{0}}, p=\frac{p^{\prime}}{p_{0}}, \quad \boldsymbol{q}=\frac{\boldsymbol{q}^{\prime}}{p_{0} v_{0}}, \mu=\frac{\mu^{\prime}}{\mu_{0}}, f=\frac{f^{\prime} v_{0}^{3}}{n_{0}},
\end{gathered}
$$

with the help of the state equation $p_{0}=n_{0} k T_{0}$. In relations (6), the dimensionless molecular velocity vector $c$ is equal to $\left(c_{p} \cos \varphi, c_{p} \sin \varphi, c_{z}\right)$.

In this study, the inverse power law potential is employed as the molecular interaction potential; therefore, viscosity can be calculated by power law temperature dependence as

$$
\mu=T^{\omega},
$$

where $\omega$ is the viscosity index, which is equal to 0.5 for Hard Sphere model and 1 for the Maxwell model [18].

Incorporating dimensionless quantities (6) into S-model kinetic equation (3), the dimensionless conservative form of governing equation is obtained

$$
\frac{\partial}{\partial t}(r f)+\frac{\partial}{\partial r}\left(r f c_{p} \cos \varphi\right)-\frac{\partial}{\partial \varphi}\left(f c_{p} \sin \varphi\right)+\frac{\partial}{\partial z}\left(r f c_{z}\right)=r \delta n T^{1-\omega}\left(f^{S}-f\right) .
$$


The above equation is subjected to the following boundary conditions. The distribution function of outgoing from the axis molecules $f^{+}$is calculated from the distribution function of incoming to the axis molecules $f^{-}$taking into account the axisymmetric condition as

$$
f_{r=0}^{+}\left(t, z, r, \varphi, c_{p}, c_{z}\right)=f_{r=0}^{-}\left(t, z, r, \pi-\varphi, c_{p}, c_{z}\right),
$$

where the superscripts + and - refer to the outgoing and incoming molecules, respectively. It is supposed that the computational domain is large enough for obtaining the equilibrium far-field. Hence, we assume that the molecules entering the computational domain are distributed according to the Maxwellian law with the parameters determined by the zero-flow at the pressure and temperature corresponding to each reservoir as follows

$$
\begin{aligned}
& f_{r=R_{L}}^{-}\left(t, z, r, \varphi, c_{p}, c_{z}\right)=f_{z=-Z_{L}}^{-}\left(t, z, r, \varphi, c_{p}, c_{z}\right)=\frac{1}{\pi^{3 / 2}} \exp \left(-c_{p}^{2}-c_{z}^{2}\right), \\
& f_{r=R_{R}}^{-}\left(t, z, r, \varphi, c_{p}, c_{z}\right)=f_{z=Z_{R}}^{-}\left(t, z, r, \varphi, c_{p}, c_{z}\right)=\frac{p_{1}}{\pi^{3 / 2}} \exp \left(-c_{p}^{2}-c_{z}^{2}\right)
\end{aligned}
$$

here $R_{L}, R_{R}$ and $Z_{R}, Z_{L}$ are the radial and axial dimensions of the left and right reservoirs, respectively.

Since the influence of the gas-wall interaction on the flow is week (see Ref. [14]), the fully diffuse scattering is implemented for the molecules reflected from both sides of the wall, which separates the two reservoirs, i.e.

$$
f_{z=0 \mp, r>1}^{+}\left(t, z, r, \varphi, c_{p}, c_{z}\right)=\frac{n_{w}^{\mp}}{\pi^{3 / 2}} \exp \left(-c_{p}^{2}-c_{z}^{2}\right),
$$

where the superscripts $\mp$ refers the left $(-)$ and the right $(+)$ sides of the wall. The unknown values of the number density at the wall surfaces $n_{w}^{\mp}$ are found from the impermeability conditions

$$
n_{w, z=0 \mp, r>1}^{\mp}(t, z, r)= \pm 4 \sqrt{\pi} \int_{0}^{\infty} \int_{0}^{\pi} \int_{0}^{\infty} c_{z} f_{z=0 \mp}^{\mp}\left(t, z, r, \varphi, c_{p}, c_{z}\right) d \mathbf{c},
$$

where $d \mathbf{c}=c_{p} d c_{p} d \varphi d c_{z}$.

The dimensionless macroscopic flow parameters are defined through the distribution function as follows

$$
\begin{aligned}
& n(t, z, r)=2 \int_{-\infty}^{\infty} \int_{0}^{\pi} \int_{0}^{\infty} f d \mathbf{c}, \quad T(t, z, r)=\frac{4}{3 n} \int_{-\infty}^{\infty} \int_{0}^{\pi} \int_{0}^{\infty} C f d \mathbf{c}, \\
& u_{r}(t, z, r)=\frac{2}{n} \int_{-\infty}^{\infty} \int_{0}^{\pi} \int_{0}^{\infty} c_{p} \cos \varphi f d \mathbf{c}, \quad u_{z}(t, z, r)=\frac{2}{n} \int_{-\infty}^{\infty} \int_{0}^{\pi} \int_{0}^{\infty} c_{z} f d \mathbf{c}, \\
& q_{r}(t, z, r)=2 \int_{-\infty}^{\infty} \int_{0}^{\pi} \int_{0}^{\infty} c_{p} \cos \varphi C f d \mathbf{c}, \quad q_{z}(t, z, r)=2 \int_{-\infty}^{\infty} \int_{0}^{\pi} \int_{0}^{\infty} c_{z} C f d(\mathbf{c}, 3)
\end{aligned}
$$


where $C=\left(c_{p} \cos \varphi-u_{r}\right)^{2}+\left(c_{p} \sin \varphi\right)^{2}+\left(c_{z}-u_{z}\right)^{2}$.

The mass flow rate is practically the most significant quantity of an orifice flow and can be calculated as

$$
\dot{M}\left(t^{\prime}\right)=2 \pi m \int_{0}^{R_{0}} n^{\prime}\left(t^{\prime}, 0, r^{\prime}\right) u_{z}^{\prime}\left(t^{\prime}, 0, r^{\prime}\right) r^{\prime} d r^{\prime} .
$$

The steady state mass flow rate into vacuum $p_{1} / p_{0}=0$ under the free molecular flow conditions $(\delta=0)$ was obtained analytically in Refs. [19], [20], [18] as

$$
\dot{M}_{f m}=\frac{R_{0}^{2} \sqrt{\pi}}{v_{0}} p_{0},
$$

and this quantity is used as reference value for the reduced mass flow rate

$$
W\left(t^{\prime}\right)=\frac{\dot{M}\left(t^{\prime}\right)}{\dot{M}_{f m}} .
$$

The dimensionless mass flow rate is obtained by substituting eqs. (6), (14), (15) into eq. (16)

$$
W(t)=4 \sqrt{\pi} \int_{0}^{1} n(t, 0, r) u_{z}(t, 0, r) r d r .
$$

Initially the upstream and downstream reservoirs, separated by a diaphragm, are maintained at the pressures $p_{0}$ and $p_{1}$, respectively, and at the same temperature $T_{0}$. At time $t=0$, just after the diaphragm opening, the mass flow rate is equal to $W=1-p_{1} / p_{0}$.

In the next sections we present the numerical approach for the solution of the kinetic equation (8).

\section{Method of solution}

Firstly, the discrete velocity method (DVM) is used to separate the continuum molecular magnitude velocity spaces $c_{p}=(0, \infty), c_{z}=(-\infty, \infty)$ in the kinetic equation (8) into discrete velocity sets $c_{p_{m}}, c_{z_{n}}$, which are taken to be the roots of Hermite polynomial. The polar angle velocity space $\varphi=[0, \pi]$ is equally discretized into set of $\varphi_{l}$. Next, the set of independent kinetic equations corresponding to discrete velocity sets $c_{p_{m}}, c_{z_{n}}$ is discretized in time and space by Finite difference method (FDM).

For each reservoir its radial and axial dimensions are taken here to be equal ( $R_{L}=Z_{L}$ and $R_{R}=Z_{R}$ ), and equal to $D_{L}$ and $D_{R}$, respectively. The influence of the dimensions $D_{L}$ and $D_{R}$ on the macroscopic parameters distribution is discussed in Section 4.6. In the physical space, the uniform grid $\left(2 N_{O} * N_{O}\right)$ with square cells is constructed near the orifice $(z=(-1,1), r=(0,1))$, where $N_{O}$ is the number of the grid points through the orifice. At the remaining computational domain $\left(z=\left(-Z_{L},-1\right) \cup\left(1, Z_{R}\right), r=\left(1, R_{L / R}\right)\right)$, the non-uniform 
158

discretization using increasing power-law of 1.05 is implemented for both radial and axial directions, as it is illustrated in Fig. 1.

The spacial derivatives are approximated by one of two upwind schemes: the first-order accurate scheme or second-order accurate TVD type scheme. The time derivative is approximated by the time-explicit Euler method. The combination of a second-order spatial discretization with forward Euler timemarching is unstable, according to a linear stability analysis [21]. However the presence of the non-linear limiter keeps it stable [21], [22]. The details of the implemented approximations are discussed in Section 4.1.

As an example the second-order accurate TVD upwind scheme with the time-explicit Euler approximation is given for the case of $\cos \varphi_{l}>0, \sin \varphi_{l}>0$ and $c_{z_{n}}>0$, when the kinetic equation (8) is replaced by the set of independent discretized equations

$$
\begin{aligned}
& \frac{r_{j} f_{i, j, l, m, n}^{k+1}-r_{j} f_{i, j, l, m, n}^{k}}{\Delta t^{k}}+c_{p_{m}} \cos \varphi_{l} \frac{F_{i, j+1 / 2, l, m, n}^{k}-F_{i, j-1 / 2, l, m, n}^{k}}{0.5\left(r_{j+1}-r_{j-1}\right)} \\
- & c_{p_{m}} \frac{f_{i, j, l+1, m, n}^{k} \sin \varphi_{l+1 / 2}-f_{i, j, l, m, n}^{k} \sin \varphi_{l-1 / 2}}{2 \sin \left(\Delta \varphi_{l} / 2\right)}+c_{z_{n}} \frac{F_{i+1 / 2, j, l, m, n}^{k}-F_{i-1 / 2, j, l, m, n}^{k}}{0.5\left(z_{i+1}-z_{i-1}\right)} \\
= & r_{j} \delta n_{i, j}^{k}\left(T_{i, j}^{k}\right)^{1-\omega}\left(\left(f_{i, j, l, m, n}^{S}\right)^{k}-f_{i, j, l, m, n}^{k}\right),
\end{aligned}
$$

where $f_{i, j, l, m, n}^{k}=f\left(t^{k}, z_{i}, r_{j}, \varphi_{l}, c_{p_{m}}, c_{z_{n}}\right), \Delta t^{k}=t^{k+1}-t^{k}, \Delta z_{i}=z_{i}-z_{i-1}$, $\Delta r_{j}=r_{j}-r_{j-1}, \Delta \varphi_{l}=\varphi_{l}-\varphi_{l-1}$. In eq. (18), the approximation of derivative of axisymmetric transport term (with respect to $\varphi$ ) is implemented with trigonometric correction [23], which helps to reduce considerably the total number of grid points $N_{\varphi}$ in the polar angle velocity space $\varphi$.

The second-order edge fluxes in the point of physical space $i, j$ are computed as

$$
F_{i \pm 1 / 2, j, l, m, n}^{k}=f_{i \pm 1 / 2, j, l, m, n}^{k} r_{j}, \quad F_{i, j \pm 1 / 2, l, m, n}^{k}=f_{i, j \pm 1 / 2, l, m, n}^{k} r_{j \pm 1 / 2}
$$

$$
\begin{aligned}
f_{i+1 / 2, j, l, m, n}^{k} & = \begin{cases}f_{i, j, l, m, n}^{k}+0.5 \Delta z_{i+1} \operatorname{minmod}\left(D_{i+1 / 2, j, l, m, n}, D_{i-1 / 2, j, l, m, n}\right) & \text { if } c_{z_{n}} \geq 0 \\
f_{i+1, j, l, m, n}^{k}-0.5 \Delta z_{i+1} \operatorname{minmod}\left(D_{i+3 / 2, j, l, m, n}, D_{i+1 / 2, j, l, m, n}\right) & \text { if } c_{z_{n}}<0,\end{cases} \\
f_{i, j+1 / 2, l, m, n}^{k} & = \begin{cases}f_{i, j, l, m, n}^{k}+0.5 \Delta r_{j+1} \operatorname{minmod}\left(D_{i, j+1 / 2, l, m, n}, D_{i, j-1 / 2, l, m, n}\right) & \text { if } \cos \varphi_{l} \geq 0 \\
f_{i, j+1, l, m, n}^{k}-0.5 \Delta r_{j+1} \operatorname{minmod}\left(D_{i, j+3 / 2, l, m, n}, D_{i, j+1 / 2, l, m, n}\right) & \text { if } \cos \varphi_{l}<0 .\end{cases}
\end{aligned}
$$

where $r_{j+1 / 2}=0.5\left(r_{j}+r_{j+1}\right)$ and

$$
D_{i+1 / 2, j, l, m, n}=\frac{f_{i+1, j, l, m, n}^{k}-f_{i, j, l, m, n}^{k}}{\Delta z_{i+1}}, \quad D_{i, j+1 / 2, l, m, n}=\frac{f_{i, j+1, l, m, n}^{k}-f_{i, j, l, m, n}^{k}}{\Delta r_{j+1}} .
$$

The slope limiter minmod introduced in [24], [21] is given by

$$
\operatorname{minmod}(a, b)=0.5(\operatorname{sign}(a)+\operatorname{sign}(b)) \min (|a|,|b|) .
$$


Table 1: Numerical grid parameters

\begin{tabular}{|c|c|c|c|}
\hline Phase space & Reservoir & \multicolumn{2}{|c|}{ Total number of points } \\
\hline Physical space $z, r$ & $\begin{array}{l}\text { Left } \\
\text { Right }\end{array}$ & $N_{O}=40$ & $\begin{array}{c}N_{z l} \times N_{r l}=96 \times 96 \\
N_{z r} \times N_{r r}=101 \times 101\end{array}$ \\
\hline Molecular velocity space $\varphi, c_{p}, c_{z}$ & Left \& Right & \multicolumn{2}{|c|}{$N_{\varphi} \times N_{c p} \times N_{c z}=40 \times 16 \times 16$} \\
\hline
\end{tabular}

The details of computational grid parameters are given in Table 1.

Concerning the temporal discretization, the time step should satisfy the classical Courant-Friedrichs-Lewy (CFL) condition [25] and must also be smaller than the mean collision time, or relaxation time, which is inverse of the collision frequency $\nu$. Consequently, the time step must satisfy the following criterion

$$
\Delta t \leq C F L / \max _{i, j, l, m, n}\left(\frac{c_{p_{m}}}{\Delta r_{j}}+\frac{c_{p_{m}}}{r_{1} \Delta \varphi_{l}}+\frac{c_{z_{n}}}{\Delta z_{i}}, \nu_{i, j}\right) .
$$

As the mass flow rate is the most important characteristic of the flow through an orifice the convergence criterion is defined for this quantity as follows

$$
\frac{\left|W\left(t^{k+1}\right)-W\left(t^{k}\right)\right|}{W\left(t^{k}\right) \Delta t^{k}} \leq \varepsilon
$$

where $\varepsilon$ is a positive number and it is taken equal to $10^{-6}$. It is to note that this convergence criterion differs from that used in Ref. [13], where the transient flow through a slit is simulated. Here we introduce the time step in the expression of the convergence criterion. It allows us to have the same convergence criterion when the size of the numerical grid in the physical space and consequently the time step according to the CFL condition (23) change. The expression of the convergence criterion (24) may be considered as the criterion on the velocity of the mass flow rate changes. The time moment, when the criterion (24) is achieved, is notified as $t_{\varepsilon}$ and the corresponding mass flow rate as $W=W\left(t_{\varepsilon}\right)$. It is to underline that the mass flow rate was chosen here as the convergence parameter, as it is the most important and useful characteristic of the flow. However the calculation were conducted in the most cases, except $p_{1} / p_{0}=0.9$ and $\delta=10$, until time equal to 40 , when the criterion (24) was already satisfied, in order to observe the steady state flow establishment in the flow field far from the orifice. The comments on the whole steady state flow field establishment are given in Section 4.3.

The numerical method is implemented as follows. First, the distribution function $f_{i, j, l, m, n}^{k+1}$ in the internal grid points at the new time step $k+1$ is calculated explicitly by eq. (18) from the data of the current time step $k$. At the boundaries, the distribution function is calculated using the boundary conditions (9) - (11). Once the distribution function is known, the values of the macroscopic parameters for the new time step are obtained by evaluating the integrals in eqs. (13). To do that, the Gauss-Hermite quadrature formulas are applied to calculate the integrals over $c_{p}, c_{z}$ spaces, while the trapezoidal 
rule is used for the approximation of the integrals over $\varphi$ space. After that, the mass flow rate for the new time step is evaluated by applying the trapezoidal rule for the integral in eq. (17). The macroscopic parameters and the mass flow rate are recorded as a function of time. This procedure is iterated until the convergence criterion (24) is met; i.e., steady flow conditions for the mass flow rate are reached.

It is to be noticed that the problem is six dimensional in the phase space: two variables in the physical space, three variables in the molecular velocity space and the time. In order to obtain the reasonable computational time the numerical code in parallelized by using the OpenMP technology. From the resolution of system (18) the velocity distribution function $f$ can be calculated at the new time level separately for each value of the molecular velocity, so these calculations are distributed among the separated processors units. The final results on the macroscopic parameters are obtained by the summation of the corresponding quantities over all processors.

The parallelization gives the opportunity to run the program code on multicore processor. To have an estimation about computational effort and speedup, the wall-clock times for executing the numerical code are recorded. The secondoder accurate TVD scheme requires 434 seconds for the first 100 time steps with 8 cores of processors AMD $84352600 \mathrm{MHz}$ and $4 \mathrm{~Gb}$ of memory for each core, whereas the first-order accurate scheme takes 242 seconds for the same task. These wall-clock times are 2585 and 1518 seconds for second-oder accurate TVD scheme and first-order accurate scheme, respectively, when only 1 core is used.

\section{Numerical results}

The numerical simulations are conducted for four values of the pressure ratio $p_{1} / p_{0}=0,0.1,0.5,0.9$ which correspond to flow into vacuum, flow of strong, medium and weak non-equilibrium. For each value of pressure ratio $p_{1} / p_{0}$ the calculations are carried out with four values of rarefaction parameter $\delta=0.1,1,10,100 ;$ i.e., from the near free molecular to hydrodynamic flow regimes.

\subsection{Different approximations of the spatial derivatives}

Two numerical schemes are implemented for the approximation of the spatial derivatives: the first-order accurate scheme and the TVD scheme with minmod slope limiter. The CFL number for both schemes was equal to 0.95. The computational time per time step by using the same computational grid is in $70 \%$ longer for TVD scheme than for the first-order accurate scheme. However, in order to reach the same uncertainty of the mass flow rate the four times larger number of grid points in each dimension of physical space is needed for the first-order accurate scheme, $N_{O}=160$, instead of 40 for the TVD scheme. Therefore all simulations are carried out by using the TVD scheme.

After the various numerical tests the optimal dimensions of the numerical grid are found (shown in Table 1), which guarantee the numerical uncertainty for 
Table 2: Dimensionless flow rate $W(17)$ vs rarefaction parameter $\delta$ and pressure ratio $p_{1} / p_{0}$. Present results $W=W\left(t_{\varepsilon}\right)$, the results from Ref. [5] $\left(W^{a}\right)$, where the steady BGK-model kinetic equation was solved using the fixed point method, the results obtained in Ref. [14] $\left(W^{b}\right)$ by the DSMC technique.

\begin{tabular}{c|ccc|cc|ccc|ccc}
\hline \multirow{2}{*}{$\delta$} & \multicolumn{3}{|c|}{$p_{1} / p_{0}=0}$. & \multicolumn{2}{c|}{0.1} & \multicolumn{3}{c|}{0.5} & \multicolumn{3}{c}{0.9} \\
\cline { 2 - 12 } & $W$ & $W^{a}$ & $W^{b}$ & $W$ & $W^{b}$ & $W$ & $W^{a}$ & $W^{b}$ & $W$ & $W^{a}$ & $W^{b}$ \\
\hline \hline 0.1 & 1.020 & 1.020 & 1.014 & 0.919 & 0.91 & 0.515 & 0.515 & 0.509 & 0.1039 & 0.105 & 0.1025 \\
1. & 1.150 & 1.152 & 1.129 & 1.054 & 1.032 & 0.636 & 0.635 & 0.613 & 0.1356 & 0.140 & 0.1297 \\
10. & 1.453 & 1.472 & 1.462 & 1.427 & 1.435 & 1.188 & 1.216 & 1.188 & 0.4009 & 0.432 & 0.4015 \\
100. & 1.527 & 1.508 & 1.534 & 1.519 & 1.524 & 1.339 & 1.325 & 1.344 & 0.6725 & 0.669 & 0.6741 \\
\hline
\end{tabular}

the mass flow rate of the order of $1 \%$. The time step, determined by eq. (23), depends crucially on the classical CFL condition subjected to the additional restriction for the time step to be smaller than the mean collision time. However, for the chosen numerical grid in the physical space, see Table 1, the latter restriction is satisfied automatically. Therefore a unique time step $\Delta t=0.1543 \times$ $10^{-4}$ is used for all the presented here cases.

\subsection{Mass flow rate}

The steady-state values of the mass flow rate $W=W\left(t_{\varepsilon}\right)$ are presented in Table 2. These values are in good agreement with the results of Refs. [5], [14], obtained from the solution of the stationary BGK-model kinetic equation by the fixed point method [5] and by applying the DSMC approach [14]. The discrepancy is less than $5 \%$ for all considered cases.

The values of mass flow rate $W(t)$ at several time moments, from $t=0$ to $\sim 40$, are given in Table 3 . The column $\left(t_{\varepsilon}\right)$ corresponds to the time needed to reach the convergence criterion (24).

To have an estimation of the computational efforts required to achieve the convergence criterion (24) the corresponding dimensionless time $t_{\varepsilon}$ and the number of the time steps are presented in Tables 3 and 4, respectively. The time evolution of the residual, defined according to eq. (24), is shown on Fig. 2 for different pressure ratios to illustrate the convergence velocity of the numerical technique. The slowest convergence rate for $p_{1} / p_{0}=0$ and 0.1 is corresponding to hydrodynamic regime, whilst that for $p_{1} / p_{0}=0.5$ and 0.9 is in slip regime. Nevertheless, the fastest convergence rate is observed at transitional regime for all pressure ratios.

The evolution of the mass flow rate $W(t)$ to its steady state value (given in Table 3) is also demonstrated in Fig. 3 for different pressure ratios. The time interval shown in Fig. 3 is restricted to the time equal to 40 even if the flow does not completely establish for this time moment in the case of pressure ratio equal to 0.9. The common behavior is observed for the pressure ratios 0 and 0.1 with relatively rapid mass flow rate establishment. It is to note that, in the hydrodynamic regime, the slope of the mass flow rate evolution reduces sharply for the both pressure ratios near the time equal to 3 whilst this slope reduction is smooth for other pressure ratios. We can observe anew the longer time of the 
steady state flow establishment for $p_{1} / p_{0}=0.9$ in whole range of the rarefaction parameter, see Fig. 3d).

In the hydrodynamic flow regime the mass flow rate has a maximum, than it decreases to reach after its steady state value from above. This tendency is visible in the hydrodynamic regime, but the same trend appears in all other regimes, though there it is less apparent because the amplitude of the mass flow rate changes is smaller. The non monotone behavior of the residual, see Fig. 2 , confirms the oscillatory character of the mass flow rate conducting in time. This behavior is related to the propagation of the initial perturbations created by the orifice opening toward the boundary of the computational domain. It is to note that the similar behavior of the mass flow rate was observed also in Ref. [11].

To characterize the mass flow rate evolution in time we introduce also the time $t_{s}$ as a last time moment when the mass flow rate differs by $1 \%$ from its steady state value $W\left(t_{\varepsilon}\right)$. The values of $t_{s}$ for various pressure ratios and the rarefaction parameters are provided in Table 3 . The two main trends for time $t_{s}$, column $\left(t_{s}\right)$ in Table 3, are found: for the pressure ratios 0, 0.1 and 0.5 the longest time to reach the steady state is needed under the transitional flow regime $(\delta=1)$, whilst for the pressure ratio 0.9 this maximum of time $t_{s}$ appears in the slip flow regime $(\delta=10)$. For the all considered pressure ratios the minimum of $t_{s}$ corresponds to the near free molecular flow regime $(\delta=0.1)$. It is to note that the exceptionally long time to steady state flow establishment is found in the case $p_{1} / p_{0}=0.9$ and $(\delta=10)$.

The time to steady state mass flow rate establishment, $t_{s}$, is compared to the corresponding quantity $t_{s}^{*}$, obtained by DSMC method in Ref. [11], see the last column of Table 3 . The values of $t_{s}^{*}$ provided in Ref. [11] are slightly smaller than those obtained in the present simulations. The largest difference between two values in more than 2 times, corresponds to the pressure ratio equal to 0.5 in the near hydrodynamic regime $(\delta=100)$, see Table 3 . It is noteworthy that due to the statistical scattering of the DSMC technique the estimation of the time to establish the steady flow is more difficult from the DSMC results than by applying the DVM method.

From dimensionless time $t_{s}$ provided in Table 3 , one can calculate easily the dimensional time $t_{s}^{\prime}$ needed to obtain the steady-state mass flow rate by using eqs. (1), (2), (6). For example, He at room temperature $T_{0}=300 \mathrm{~K}$ has the most probable molecular speed $v_{0}=1116.05 \mathrm{~m} / \mathrm{s}$ and viscosity coefficient $\mu_{0}=1.985 \times 10^{-5} \mathrm{Nsm}^{-2}$ (provided in [18]) . If one consider an orifice of the radius $R_{0}=0.5 \mathrm{~mm}$ and pressure in the upstream reservoir $p_{0}=44.31 \mathrm{~Pa}$, the gas flow is in transitional regime $(\delta=1$.). The dimensionless time of the expansion into vacuum (case $p_{1} / p_{0}=0$ in Table 3 ) is equal to 6.95 and the corresponding dimensional time is $3.11 \mu \mathrm{s}$.

The mass flow rate as a function of time was fitted using the following model

$$
W(t)=W_{t=0}+\left(W_{t=t_{\varepsilon}}-W_{t=0}\right)(1-\exp (-t / \tau)),
$$

where the value at the time moment $t=0$ is calculated as $W_{t=0}=1-p_{1} / p_{0}$ and $W_{t=t_{\varepsilon}}$ is the value of the mass flow rate corresponding to the time moment $t=t_{\varepsilon}$ 
Table 3: Mass flow rate $W$ for different time moments. The time $t_{s}$ of the steady state flow establishment as a function of the rarefaction parameter $\delta$ and the pressure ratio $p_{1} / p_{0} ; t_{s}^{*}$ corresponds to the data from Ref. [11]. Time $t_{s}$ is here the dimensionless value, obtained using eqs. (2) and (6).

\begin{tabular}{|c|c|c|c|c|c|c|c|c|c|c|}
\hline \multirow{2}{*}{$p_{1} / p_{0}$} & \multirow{2}{*}{$\delta$} & \multicolumn{6}{|c|}{$W$} & \multirow[b]{2}{*}{$t_{\varepsilon}$} & \multirow[b]{2}{*}{$t_{s}$} & \multirow{2}{*}{$t_{s}^{*}$} \\
\hline & & $t=0$ & 1 & 5 & 10 & 20 & 40 & & & \\
\hline \multirow{4}{*}{0.} & 0.1 & 1.0 & 1.003 & 1.016 & 1.019 & 1.020 & 1.020 & 19.71 & 2.35 & \\
\hline & 1. & 1.0 & 1.028 & 1.126 & 1.146 & 1.149 & 1.148 & 15.77 & 6.95 & \\
\hline & 10. & 1.0 & 1.120 & 1.423 & 1.455 & 1.453 & 1.456 & 20.60 & 6.15 & \\
\hline & 100. & 1.0 & 1.171 & 1.511 & 1.528 & 1.522 & 1.527 & 35.26 & 5.07 & \\
\hline \multirow{4}{*}{0.1} & 0.1 & 0.9 & 0.903 & 0.915 & 0.918 & 0.919 & 0.919 & 20.48 & 2.61 & \\
\hline & 1. & 0.9 & 0.928 & 1.027 & 1.049 & 1.054 & 1.053 & 16.87 & 7.84 & 6.4 \\
\hline & 10. & 0.9 & 1.035 & 1.383 & 1.427 & 1.427 & 1.429 & 20.94 & 7.05 & 7.3 \\
\hline & 100. & 0.9 & 1.145 & 1.500 & 1.520 & 1.514 & 1.519 & 26.94 & 5.24 & 4.4 \\
\hline \multirow{4}{*}{0.5} & 0.1 & 0.5 & $\begin{array}{l}0.503 \\
\end{array}$ & 0.512 & 0.514 & 0.515 & 0.515 & 22.39 & 3.64 & \\
\hline & 1. & 0.5 & 0.523 & 0.607 & 0.629 & 0.635 & 0.635 & 18.76 & 9.91 & 8.7 \\
\hline & 10. & 0.5 & 0.623 & 1.060 & 1.177 & 1.191 & 1.189 & 26.94 & 9.84 & 9.1 \\
\hline & 100. & 0.5 & 0.756 & 1.309 & 1.351 & 1.339 & 1.342 & 23.59 & 5.54 & 14.0 \\
\hline \multirow{4}{*}{0.9} & 0.1 & 0.1 & 0.1007 & 0.1030 & 0.1036 & 0.1039 & 0.1039 & 22.82 & 4.44 & \\
\hline & 1. & 0.1 & 0.1059 & 0.1275 & 0.1338 & 0.1356 & 0.1354 & 19.20 & 10.83 & \\
\hline & 10. & 0.1 & 0.1292 & 0.2602 & 0.3396 & 0.3893 & 0.3991 & 152.6 & 30.37 & \\
\hline & 100. & 0.1 & 0.1571 & 0.4248 & 0.6222 & 0.6943 & 0.6727 & 36.10 & 12.28 & \\
\hline
\end{tabular}

Table 4: Number of time steps to satisfy convergence criterion (24)

\begin{tabular}{c|c|c|c|c}
\hline \multirow{2}{*}{$\delta$} & \multicolumn{4}{|c}{ Total number of time steps $N(\times 100)$} \\
\cline { 2 - 5 } & $p_{1} / p_{0}=0$. & 0.1 & 0.5 & 0.9 \\
\hline \hline 0.1 & 1277 & 1327 & 1451 & 1479 \\
1. & 1022 & 1093 & 1216 & 1244 \\
10. & 1335 & 1357 & 1746 & 9889 \\
100. & 2285 & 1746 & 1529 & 1339 \\
\hline
\end{tabular}


311

312

313

314

315

316

317

318

319

320

321

322

323

324 order of $0.3 \%$ for the same pressure ratio and $\delta=1$. these values become larger, see Table 5 .

where the convergence criterion (24) is achieved. Both the values are given in Tables 2 and 3. The fitting parameter (characteristic time) $\tau$ for various pressure ratios and rarefaction parameters are provided in Table 5 with the corresponding uncertainty. It is to note that very similar values of $\tau$ are found for the pressure ratios 0 and 0.1 for all rarefaction range. For the pressure ratios 0.5 and 0.9 and for the high level of gas rarefaction also the similar values of the fitting parameter $\tau$ are found. However in the slip and hydrodynamic flow regimes

Figure 4 demonstrates that the exponential representation in form of eq. (25) gives the good estimation for the time evolution of the mass flow rate. The coefficient of determination $R^{2}$ of the fitting curve is equal, for example, to 0.990 for the case $p_{1} / p_{0}=0.9$ and $\delta=1$ and decreases to 0.973 for $p_{1} / p_{0}=0.9$ and $\delta=100$. The maximal difference between the values of the mass flow rate, given by the fitting curve and by the numerical solution of the S-model kinetic equation, is less than $5 \%$ for the case $p_{1} / p_{0}=0.9$ and $\delta=100$ and it is of the

Table 5: Characteristic time $\tau$ with $99 \%$ confidence interval obtained from fit model eq. (25)

\begin{tabular}{c|c|c|c|c}
\hline \multirow{2}{*}{$\delta$} & \multicolumn{4}{|c}{ Characteristic time $\tau$} \\
\cline { 2 - 5 } & $p_{1} / p_{0}=0$. & 0.1 & 0.5 & 0.9 \\
\hline \hline 0.1 & $3.415 \pm 0.029$ & $3.484 \pm 0.028$ & $3.546 \pm 0.024$ & $3.561 \pm 0.023$ \\
1. & $2.940 \pm 0.034$ & $3.112 \pm 0.031$ & $3.429 \pm 0.028$ & $3.551 \pm 0.028$ \\
10. & $2.286 \pm 0.032$ & $2.393 \pm 0.030$ & $3.269 \pm 0.039$ & $6.459 \pm 0.013$ \\
100. & $1.879 \pm 0.032$ & $1.731 \pm 0.019$ & $2.072 \pm 0.037$ & $5.597 \pm 0.083$ \\
\hline
\end{tabular}

\subsection{Flow field}

After the diaphragm opening the gas starts to flow toward the downstream reservoir. However, even in the upstream reservoir the flow field becomes perturbed from its initial state. From the near free molecular $(\delta=0)$ to the slip flow regime $(\delta=10)$ for all considered pressure ratios $p_{1} / p_{0}=0-0.9$ the time behavior of the macroscopic parameters are very similar. The two typical examples of the macroscopic parameters variation in time are presented in Fig. 5 for the cases $p_{1} / p_{0}=0.1$ and 0.5 and $\delta=1$. The number density $n$ smoothly changes from its value in the upstream reservoir to its downstream value. The temperature drops through the orifice due to the flow acceleration and increases up to its initial value far from the orifice in the downstream reservoir. The temperature drop is larger for the smaller values of the pressure ratio: the temperature decreasing just after the orifice is of the order of $25 \%$ for $p_{1} / p_{0}=0.1$ and $\delta=1$ and it becomes very small (less than 1\%) when the pressure ratio increases up to 0.9. The macroscopic flow velocity increases through the orifice and its rise depends also on the pressure ratio: for the smaller value of the pressure ratio the flow acceleration is higher. Far from the orifice in the upstream and downstream reservoirs the flow velocity goes down to zero. It is to note that for the larger pressure ratio $p_{1} / p_{0}=0.9$ even in the case of the 
near hydrodynamic flow regimes, $\delta=100$, the time dependent behaviors of the macroscopic parameters are similar to the previously described.

The results obtained in Ref. [26] by using the DSMC technique are also shown in Fig. 5. The provided here DSMC data correspond to the steady state solution. Its is clear that the both techniques give very similar results. Only the temperature behaviors for $p_{1} / p_{0}=0.5$ are slightly different which can be related to the influence of the boundary conditions in the downstream reservoir.

\subsection{Near hydrodynamic regime}

Completely different behavior is observed for the all considered pressure ratios, except the case of $p_{1} / p_{0}=0.9$, in the near hydrodynamic flow regime $(\delta=100)$. For the pressure ratio $p_{1} / p_{0}=0.5$, see Fig. 6 , the shock wave appears in the right reservoir and it moves toward the downstream boundary.

For the pressure ratio $\left(p_{1} / p_{0}=0.1\right)$ the particular flow behavior is observed: the spatial cell structure of axisymmetric mildly under-expanded jet appears, formed by the system of incident and reflected shock and compression waves, see Fig. 7. The distribution of the macroscopic flow parameters for this case is shown on Fig. 8. In contrast with the previous case, the first cell shock structure does not move and the second shock wave forms after the first one with time. The shock wave position may be determined by the maximum of the number density gradient which is located at $z_{M} / R_{0}=4.31$. This position can be estimated also from the empirical relation [27]

$$
z_{M} / R_{0}=1.34 \sqrt{p_{0} / p_{1}},
$$

which predicts the Mach disk location at $z_{M} / R_{0}=4.24$ from the orifice, so very good agreement is found between the numerical result and empirical relation (26).

The streamlines for the case $p_{1} / p_{0}=0.1$ are provided in Fig. 9. It can be seen that the flow field is non symmetric and that the streamlines are not parallel to the axis of symmetry.

In the case of the gas expansion into vacuum $\left(p_{1} / p_{0}=0\right)$ the shock wave does not appear any more. Expression (26) predicts also that the shock wave position tends to infinity $\left(z_{M} / R_{0} \rightarrow \infty\right)$. In this case the flow velocity reach its maximal value, which depends only on the gas temperature in the inlet reservoir. Under the hypothesis of the adiabatic expansion and the energy conservation the following expression for the macroscopic velocity was obtained in Ref. [28]:

$$
u_{z_{\max }}=\sqrt{\frac{5 k T_{0}}{m}} .
$$

The numerical value of the maximal macroscopic velocity is equal to 1.588 which is very close to that predicted by eq. (27).

\subsection{Chocked conditions}

It is well known that a chocked flow is a limiting condition which occurs when the mass flow rate will not increase with a further decrease in the downstream pressure environment while upstream pressure is fixed [29]. Under the 
conditions $p_{1} / p_{0}<\left(p_{1} / p_{0}\right)_{*}$, where $\left(p_{1} / p_{0}\right)_{*}$ is the critical pressure ratio, the further decrease in the downstream pressure reservoir does not lead to the increase of the mass flow rate and the flow becomes "chocked". However, for the case of the flow through a thin orifice the flow never becomes "chocked". For the first time it was discovered in Ref. [30] in the case of the flow through a thin, square-edged orifice. Finally the physical point at which the choking occurs for adiabatic conditions is that the exit plane velocity is at sonic conditions. But in the case of the thin orifice flow the "sonic surface" has a convex shape and located in the downstream reservoir, see Fig. 10, where two cases of the pressure ratio $p_{1} / p_{0}=0$ and 0.1 are shown. Therefore the flow is not sonic through the orifice and it does not becomes really chocked: the mass flow rate continue to increases when pressure ratio decreases, see Table 3 and Fig. 11, especially for the low values of the rarefaction parameter. The evaluation in time of the temperature and Mach number profiles in the orifice section are shown on Fig. 12 , where one can see that the flow remains subsonic through the orifice with the maximum velocity near the orifice wall.

\subsection{Influence of the computational domain dimensions}

The study of an influence of the computational domain dimensions on the numerical results is carried out and the optimal dimensions of the left and right reservoirs are found as $D_{L}=8$ and $D_{R}=10$, respectively.

Fig. 13 shows the comparison of the macroscopic profiles evolution in time along the symmetrical axis for $p_{1} / p_{0}=0.1$ and $\delta=100$ obtained for two sizes of the downstream reservoir $D_{R}=10$ and 20. It is clear from these results that the both solutions coincide until distance $z / R_{0} \sim 8$ from the orifice and that the mass flow rate evolution is not affected at all by the dimension of the right computational domain. It is interesting to note that in the case of the flow through a slit much more larger computational domain must be chosen to obtain the numerical solution independent from the size of the computational domain.

\section{Conclusion}

Transient flow of rarefied gas through an orifice is studied on the basis of nonlinear S-model kinetic equation. The simulations are conducted from the free molecular to hydrodynamic regimes for four values of pressure ratio between reservoirs. The mass flow rate evolution in time is analyzed and it is found that the time to reach the steady state mass flow rate depends essentially on the pressure ratio between the reservoirs and on the gas flow regime in the left reservoir. It needs from 2.35 to 30.37 characteristic times to obtain the steady state mass flow rate, the maximal time to reach the steady state is found in the slip regime for the largest pressure ratio 0.9. The simple fitting formula for the time dependence of the mass flow rate is proposed. It is shown numerically that the flow through the thin orifice never becomes really chocked. 


\section{Acknowledgment}

This work was granted access to the HPC resources of Aix-Marseille Université financed by the project Equip@Meso (ANR-10-EQPX-29-01) of the program "Investissements d'Avenir" supervised by the Agence Nationale pour la Recherche. The authors thank Y. Jobic for ensuring the perfect performance of the cluster at IUSTI Laboratory. We thank Prof. F. Sharipov for providing the DSMC results from Ref. [11].

[1] K. Jousten, S. Pantazis, J. Buthing, R. Model, M. Wuest, A standard to test the dynamics of vacuum gauges in the millisecond range, Vacuum 100 (2014) 14-17.

[2] T. Lilly, S. Gimelshein, A. Ketsdever, G. N. Markelov, Measurements and computations of mass flow and momentum flux through short tubes in rarefied gases, Phys. Fluids 18 (9) (2006) 093601.1-11.

[3] S. Varoutis, D. Valougeorgis, F. Sharipov, Simulation of gas flow through tubes of finite length over the whole range of rarefaction for various pressure drop ratios, J. Vac. Sci. Technol. A. 22 (6) (2009) 1377-1391.

[4] I. Graur, A. P. Polikarpov, F. Sharipov, Numerical modelling of rarefied gas flow through a slit at arbitrary gas pressure ratio based on the kinetic equations, ZAMP 63 (3) (2012) 503-520.

[5] S. Misdanitis, S. Pantazis, D. Valougeorgis, Pressure driven rarefied gas flow through a slit and an orifice, Vacuum 86 (11) (2012) 1701 - 1708.

[6] V. V. Aristov, A. A. Frolova, S. A. Zabelok, R. R. Arslanbekov, V. I. Kolobov, Simulations of pressure-driven flows through channels and pipes with unified flow solver, Vacuum 86 SI (11) (2012) 1717-1724.

[7] V. A. Titarev, E. M. Shakhov, Computational study of a rarefied gas flow through a long circular pipe into vacuum, Vacuum 86 (11) (2012) 17091716 .

[8] V. A. Titarev, Rarefied gas flow in a circular pipe of finite length, Vacuum 94 (2013) 92-103.

[9] V. Titarev, E. Shakhov, S. Utyuzhnikov, Rarefied gas flow through a diverging conical pipe into vacuum, Vacuum 101 (0) (2014) 10 - 17.

[10] F. Sharipov, Transient flow of rarefied gas through an orifice, J. Vac. Sci. Technol. A 30 (2) (2012) 021602.1-5.

[11] F. Sharipov, Transient flow of rarefied gas through a short tube, Vacuum 90 (2013) 25-30.

[12] J. Lihnaropoulos, D. Valougeorgis, Unsteady vacuum gas flow in cylindrical tubes, Fusion Engineering and Design 86 (2011) 2139-2142. 
[13] A. Polikarpov, I. Graur, Unsteady rarefied gas flow through a slit, Vacuum 101 (2014) 79 - 85. doi:http://dx.doi.org/10.1016/j.vacuum.2013.07.006.

[14] F. Sharipov, Numerical simulation of rarefied gas flow through a thin orifice, Journal of Fluid Mechanics 518 (2004) 35-60.

[15] E. M. Shakhov, Generalization of the Krook kinetic relaxation equation, Fluid Dyn. 3 (5) (1968) 95-96.

[16] I. N. Larina, V. A. Rykov, A numerical method for calculationg axisymmetric rarefied gas flows, Comput. Math. Math. Phys. 38 (8) (1998) 1335-1346.

[17] I. Graur, M. T. Ho, M. Wuest, Simulation of the transient heat transfer between two coaxial cylinders, Journal of Vacuum Science \& Technology A: Vacuum, Surfaces, and Films 31 (6) (2013) 061603.1-9.

[18] G. A. Bird, Molecular Gas Dynamics and the Direct Simulation of Gas Flows, Oxford Science Publications, Oxford University Press Inc., New York, 1994.

[19] R. Narasimha, Orifice flow of high Knudsen numbers, J. Fluid Mech. 10 (1961) 371-384.

[20] D. R. Willis, Mass flow through a circular orifice and a two-dimensional slit at high Knudsen numbers., J. Fluid Mech. 21 (1965) 21-31.

[21] V. P. Kolgan, Application of the principle of minimizing the derivative to the construction of finite-difference schemes for computing discontinuous solutions of gas dynamics, Journal of Computational Physics 230 (7) (2011) $2384-2390$

[22] B. Van Leer, A historical oversight: Vladimir P. Kolgan and his highresolution scheme, Journal of Computational Physics 230 (7) (2011) 23872383.

[23] L. Mieussens, Discrete-velocity models and numerical schemes for the Boltzmann-bgk equation in plane and axisymmetric geometries, J. Comput. Phys. 162 (2) (2000) 429-466.

[24] P. L. Roe, Characteristic-based schemes for the euler equations, Ann. Rev. Fluid Mech 18 (1986) 337-365.

[25] R. Courant, K. Friedrichs, H. Lewy, On the partial difference equations of mathematicalphysics, IBM Journal on Research and development 11 (2) (1967) 215-234.

[26] F. Sharipov, Benchmark problems in rarefied gas dynamics, Vacuum 86 (2012) 1697-1700. 
[27] H. Ashkenas, F. S. Sherman, The structure and utilization of supersonic free jets in low density wind tunnels, in: Proceedings of the 4th International Symposium on RGD, Vol. 2, 1964, pp. 84-105.

[28] M. D. Morse, Experimental methods in the physical sciences, Vol. 29B, Academic Press Inc., 1996, Ch. Supersonic beam sources, pp. 21-47.

[29] L. Driskell, Control-valve selection and sizing, Research Triangle Park, N.C. : Instrument Society of America, 1983.

[30] R. G. Cunningham, Orifice meters with supercritical compressible flow, Transactions of the ASME 73 (1951) 625-638.

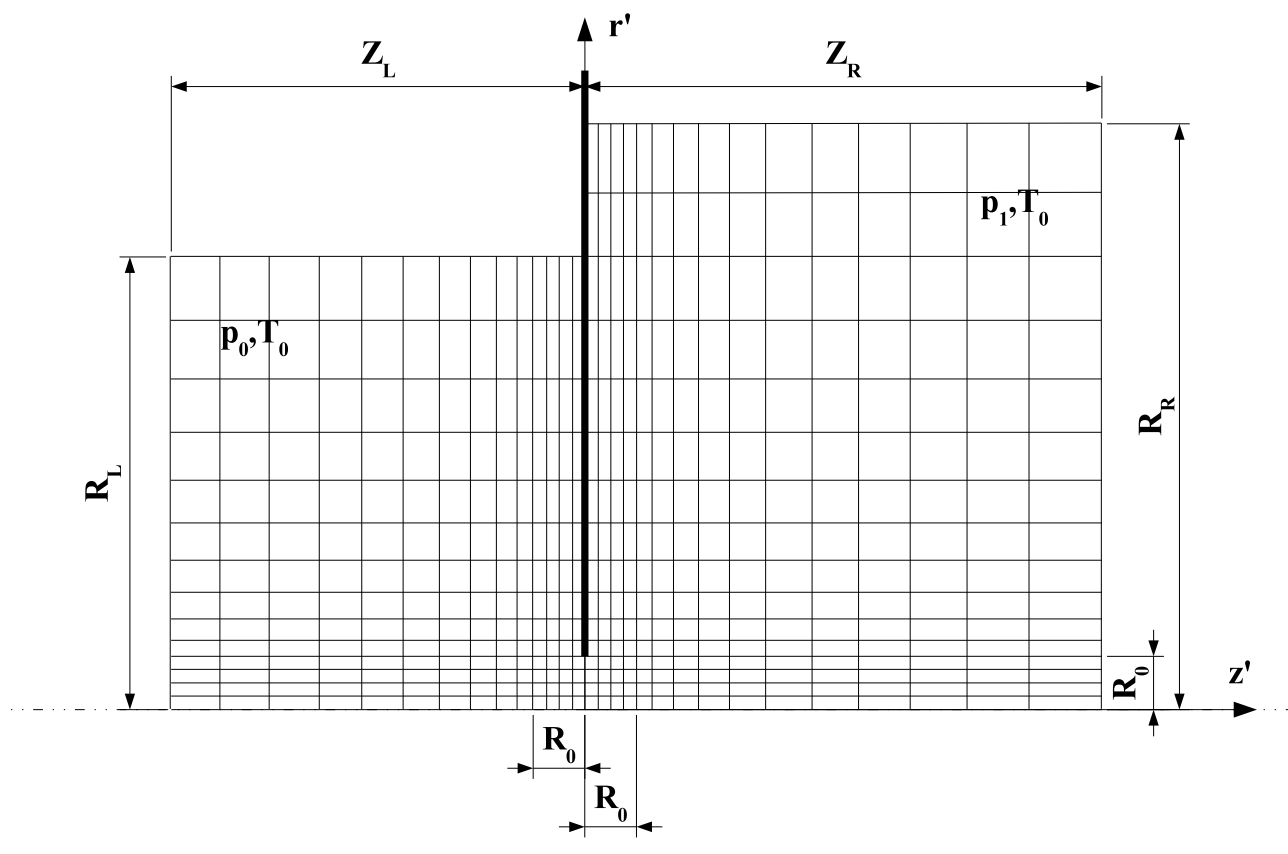

Figure 1: Lateral section and computational domain of the flow configuration 

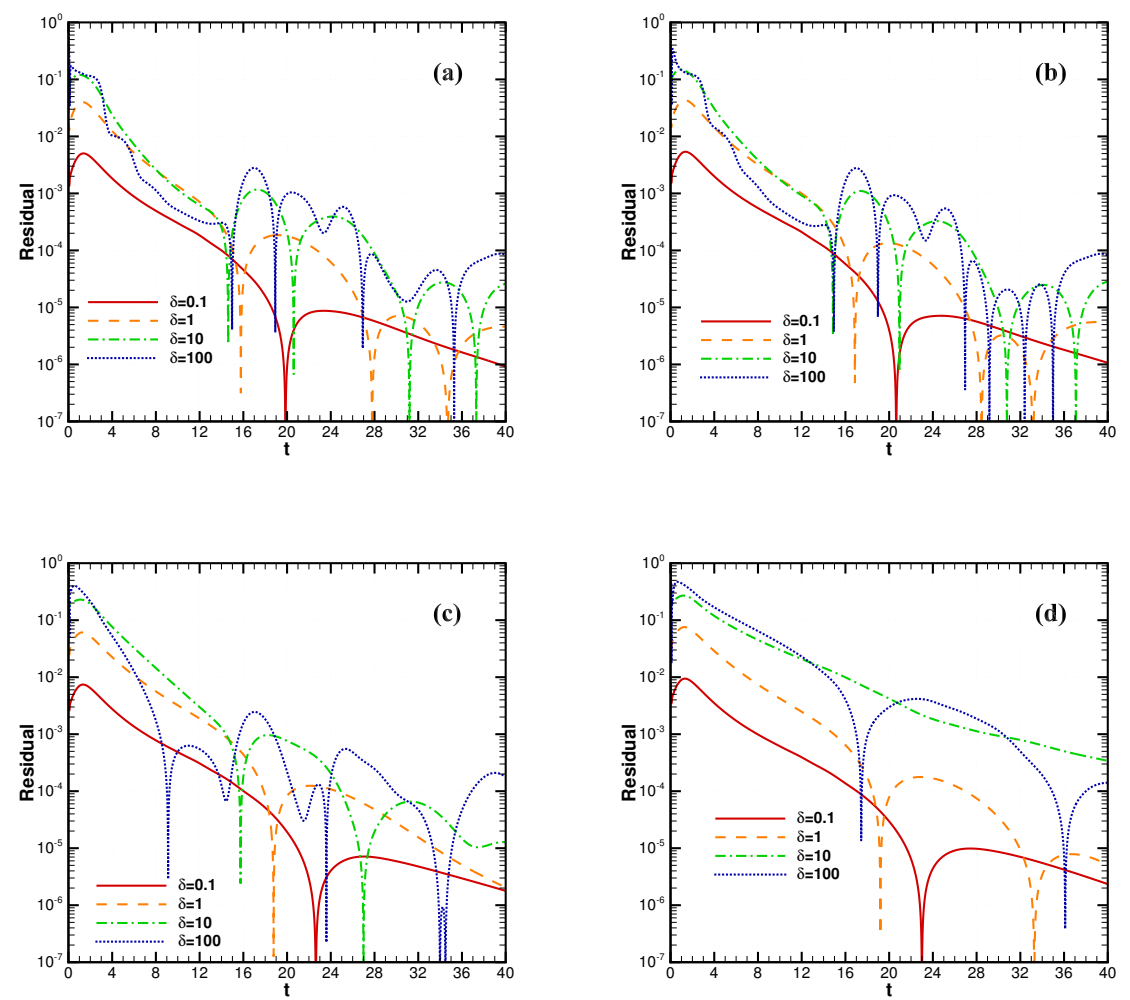

Figure 2: The time evolution of residual for $p_{1} / p_{0}=0$. (a), $p_{1} / p_{0}=0.1$ (b), $p_{1} / p_{0}=0.5$ (c), $p_{1} / p_{0}=0.9$ (d) 

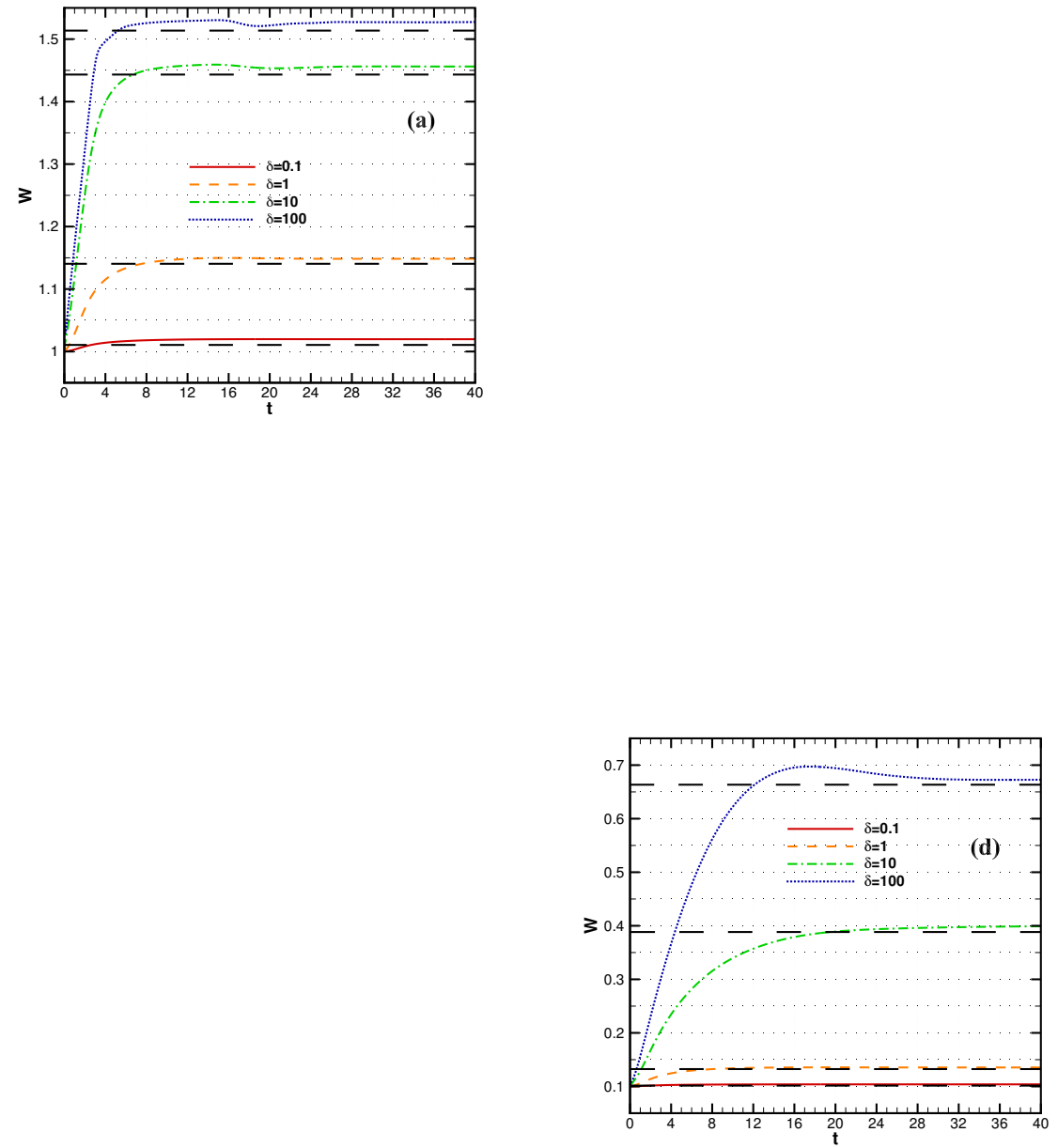

Figure 3: The time evolution of mass flow rate $W$ (solid line) and steady state solution (dashed line) for $p_{1} / p_{0}=0$. (a), $p_{1} / p_{0}=0.1$ (b), $p_{1} / p_{0}=0.5$ (c), $p_{1} / p_{0}=0.9$ (d) 


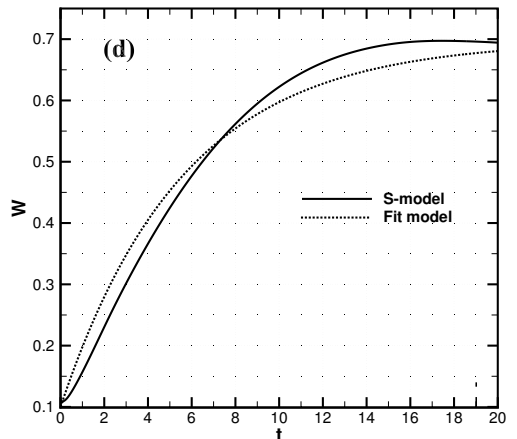

Figure 4: The time evolution of mass flow rate $W$ (solid line) obtained from S-model and fit model eq. (25) for $p_{1} / p_{0}=0 ., \delta=1$. (a), $p_{1} / p_{0}=0 ., \delta=100$. (b), $p_{1} / p_{0}=0.9, \delta=1$. (c), $p_{1} / p_{0}=0.9, \delta=100$. (d) 

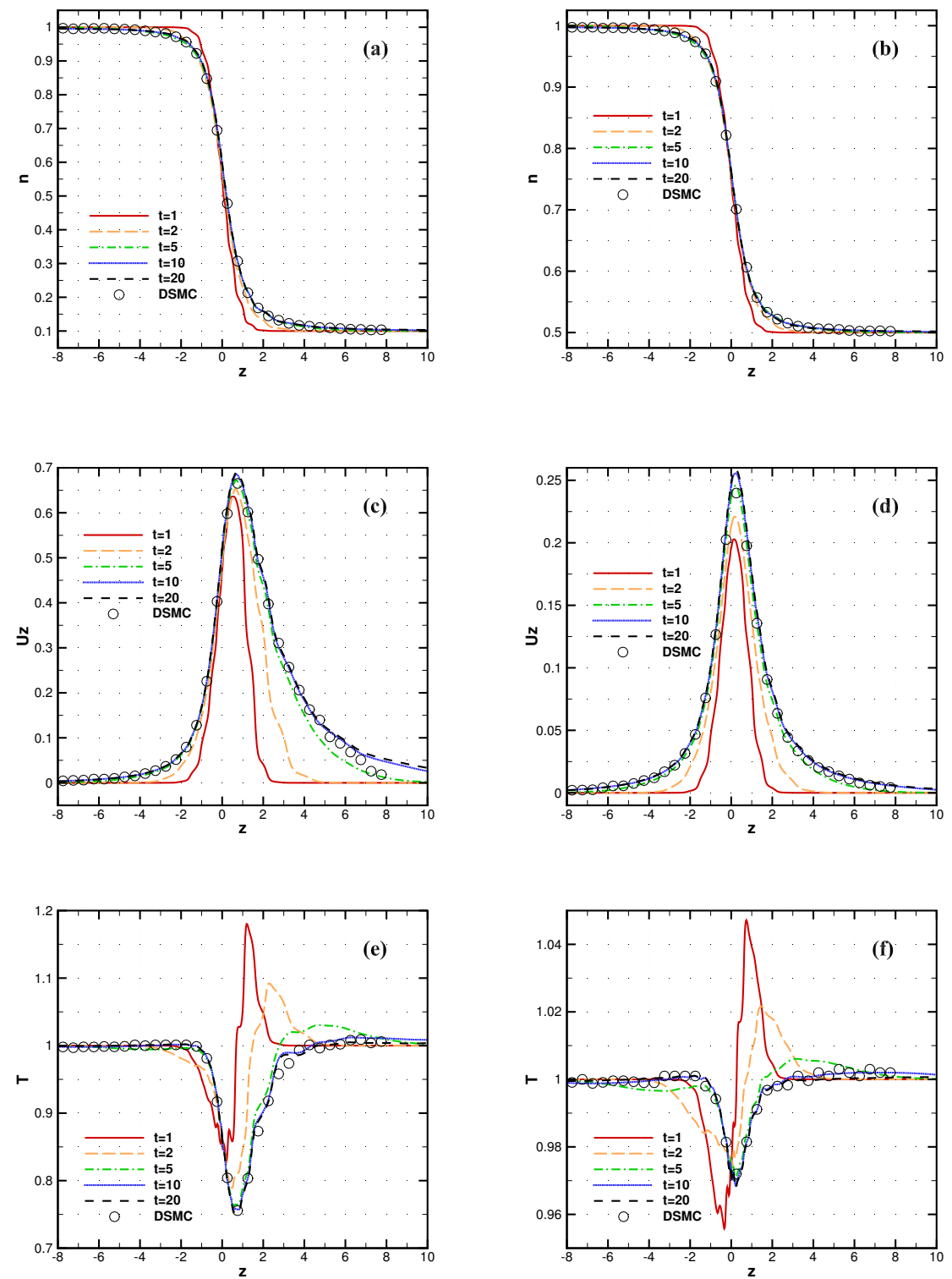

Figure 5: Distribution of density number $(\mathrm{a}, \mathrm{b})$, axial velocity $(\mathrm{c}, \mathrm{d})$, temperature $(\mathrm{e}, \mathrm{f})$ along the axis at several time moments for $p_{1} / p_{0}=0.1, \delta=1$. (a,c,e) and $p_{1} / p_{0}=0.5, \delta=1$. (b,d,f). The hollow circles correspond to the results obtained in [10] by DSMC method. 

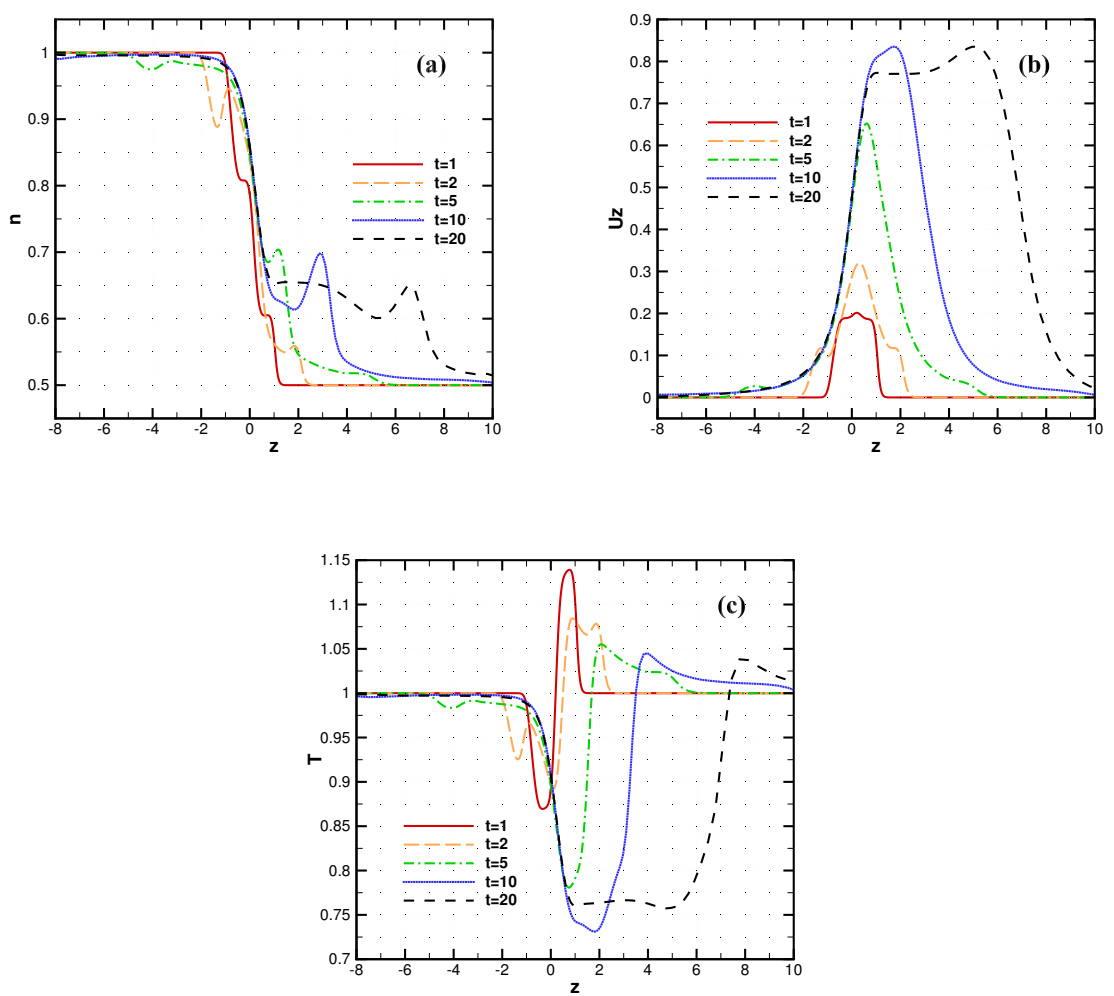

Figure 6: Distribution of density number (a), axial velocity (b), temperature (c) along the axis at several time moments for $p_{1} / p_{0}=0.5, \delta=100$. 

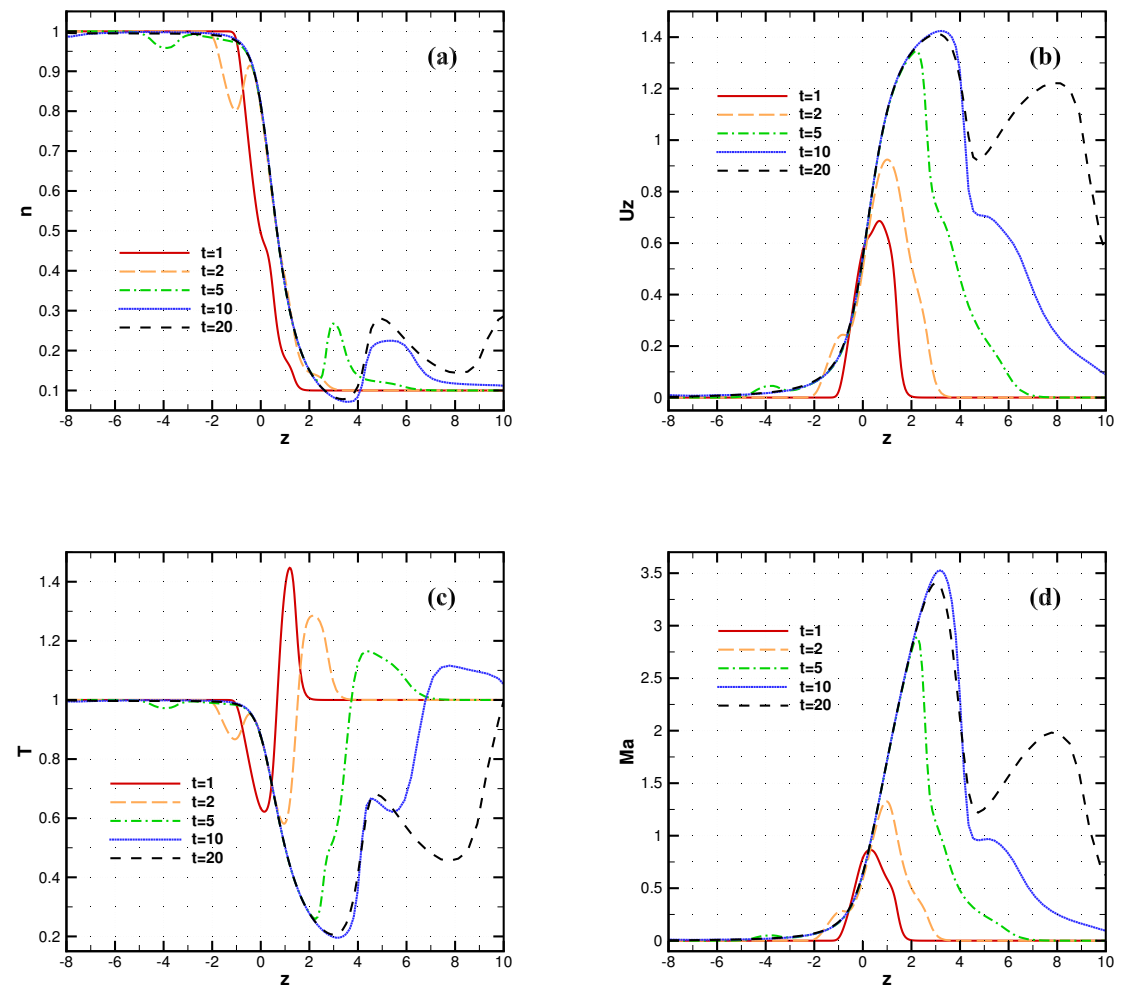

Figure 8: Distribution of density number (a), axial velocity (b), temperature (c), Mach number (d) along the axis at several time moments for $p_{1} / p_{0}=0.1, \delta=100$. 


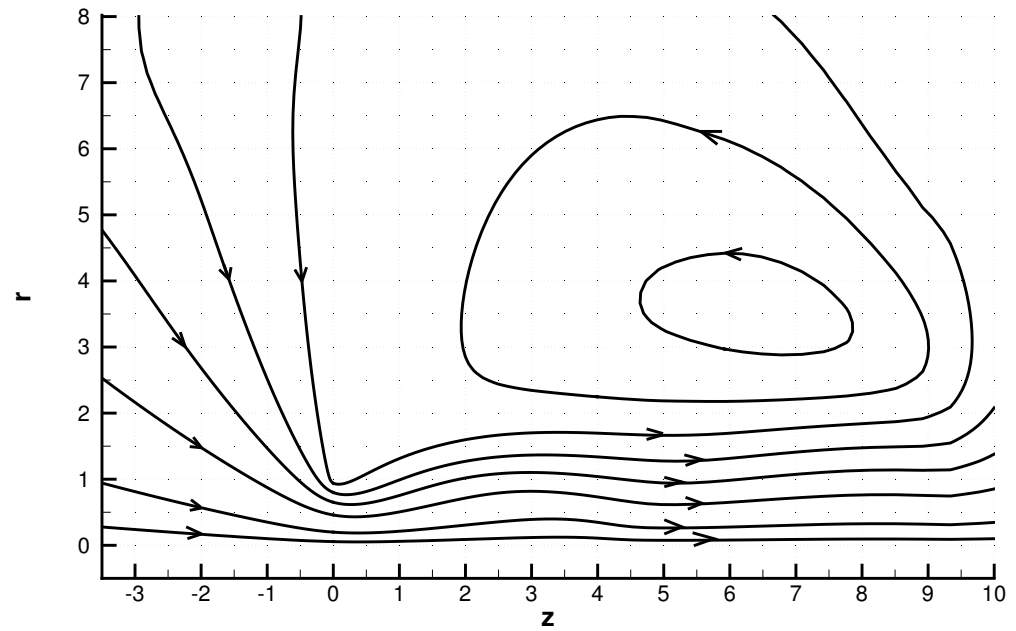

Figure 9: Stream lines at time moment $t=20$ for $p_{1} / p_{0}=0.1, \delta=100$. 

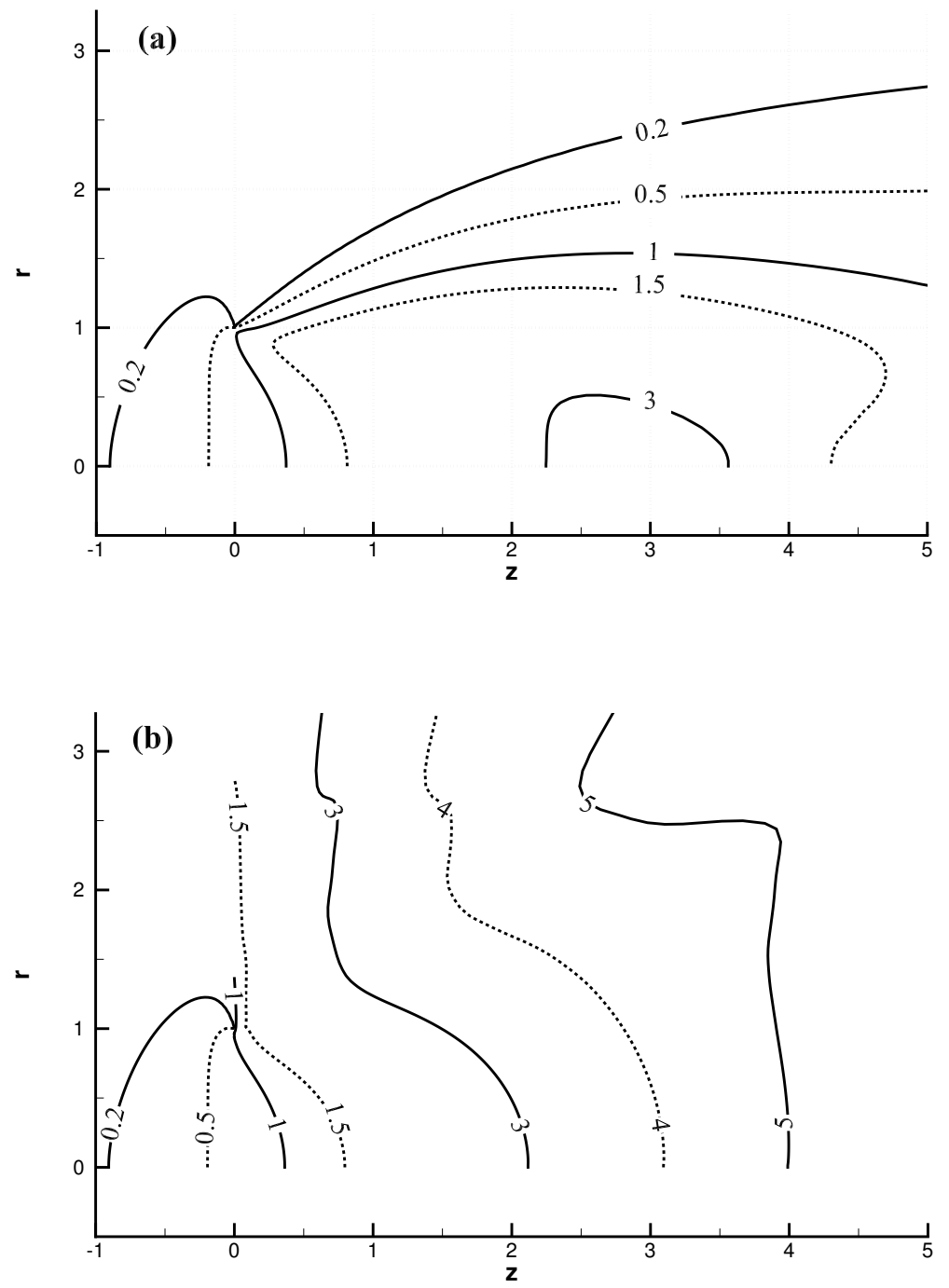

Figure 10: Mach number iso-lines at time moment $t=20$ for $p_{1} / p_{0}=0.1, \delta=100$. (a), $p_{1} / p_{0}=0 ., \delta=100$. (b) 
Figure 11: Dimensionless mass flow rate as a function of pressure ratio $p_{1} / p_{0}$ at different rarefaction parameters $\delta$ 

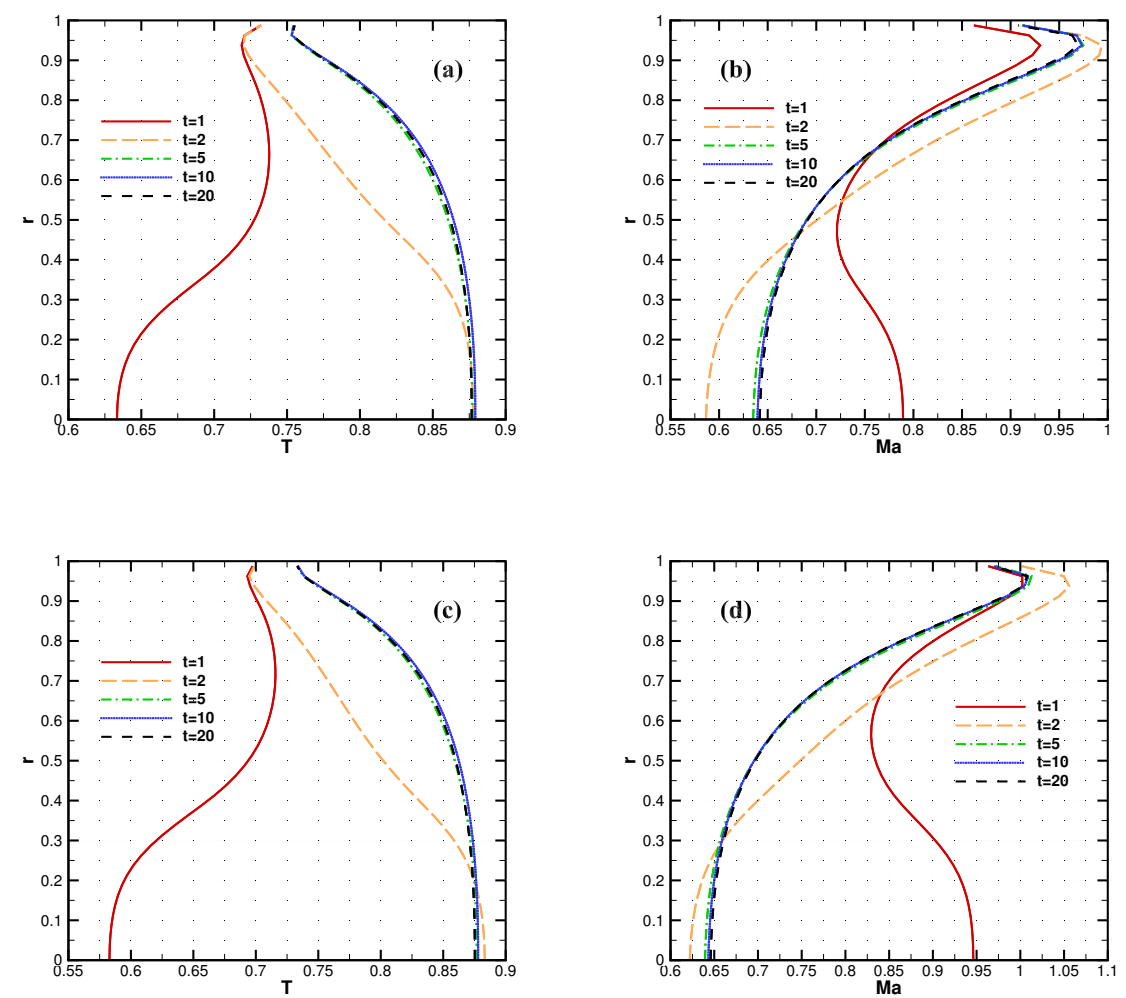

Figure 12: Distribution of temperature (a,c), Mach number (b,d) along the orifice at several time moments for $p_{1} / p_{0}=0.1, \delta=100$. (a,b), $p_{1} / p_{0}=0 ., \delta=100$. (c,d) 

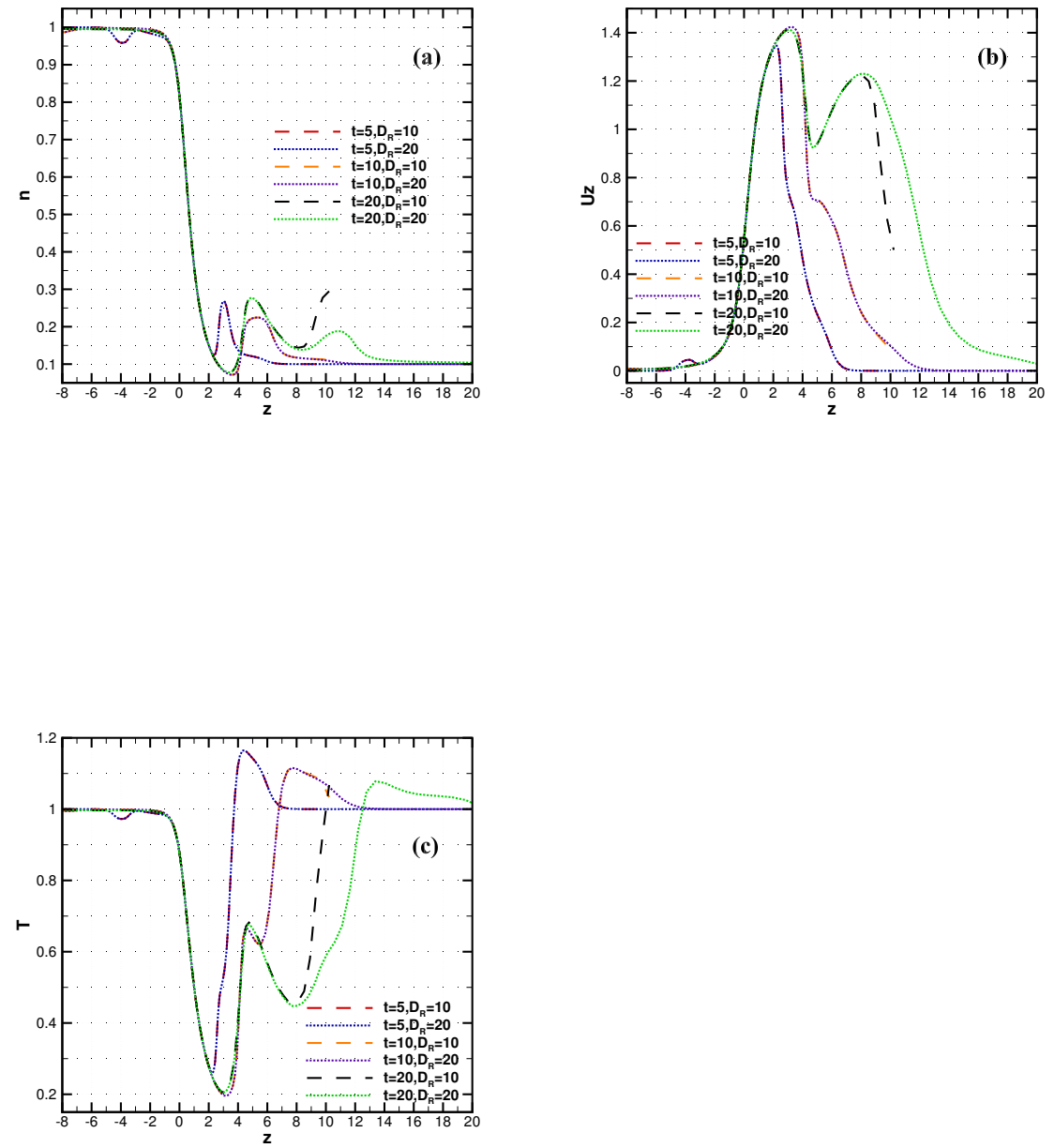

Figure 13: Distribution of density number (a), axial velocity (b), temperature (c) along the axis at time moments $t=5,10,20$ for $p_{1} / p_{0}=0.1, \delta=100$. with different computational domain sizes $D_{R}=10,20$. The time evolution of mass flow rate $W$ with different computational domain sizes $D_{R}=10,20$ (d) 